\title{
Uptake of testing for BRCA1/2 mutations in South East Scotland
}

\author{
Susan M Holloway*,1, Birgitta Bernhard ${ }^{1}$, Harry Campbell ${ }^{2}$ and Wayne WK Lam ${ }^{1}$ \\ ${ }^{1}$ Department of Clinical Genetics, Western General Hospital, Edinburgh, UK; ${ }^{2}$ Department of Public Health Sciences, \\ University of Edinburgh Medical School, Edinburgh, UK
}

We investigated the uptake of genetic testing by 54 families in South East Scotland with a BRCA1/2 mutation. At a median of 37 months since identification of the mutation, the overall rate of uptake of testing in 269 eligible family members was $32 \%$. First-degree relatives were significantly $(P<0.05)$ less likely to be referred for genetic counselling in more, compared to less, socioeconomically deprived families (46 versus $68 \%$ ). Among relatives who attended for genetic counselling, females were more likely to be tested than males (76 versus $53 \% ; P<0.05)$ and relatives with children more than those without children (82 versus $53 \% ; P<0.001$ ). Tested relatives were older than relatives who did not undergo testing (mean 41.9 versus 36.8 years, $P<0.05$ ) but did not differ in degree of relationship to the index case or in socioeconomic deprivation. Our results confirm the findings from other studies of substantially lower rates of uptake of genetic testing for BRCA1/2 mutations than anticipated in earlier predictions. Relatives in more socioeconomically deprived families were less likely to be referred for genetic counselling, which is a matter of concern. This may be partly the result of a lack of understanding of the testing process. Cascading currently does not work in breast cancer families and further work is required to investigate intrafamilial communication patterns, testing behaviour and counselling strategies. European Journal of Human Genetics (2008) 16, 906-912; doi:10.1038/ejhg.2008.17; published online 20 February 2008

Keywords: BRCA1; BRCA2; genetic testing; uptake; Scotland

\section{Introduction}

The identification of a BRCA1/2 mutation in a family with breast/ovarian cancer offers at-risk relatives the opportunity to undergo pre-symptomatic genetic testing. Gene mutation carriers may decide to undergo regular clinical surveillance, prophylactic mastectomy or oophorectomy.

Several studies ${ }^{1-3}$ have shown a high percentage ( $\left.>80 \%\right)$ of relatives to be interested in the possibility of genetic testing. In practice, uptake is low and depends on the population studied and the family history. The overall uptake of genetic testing by at-risk relatives varies from 21

*Correspondence: Dr SM Holloway, Department of Clinical Genetics, Western General Hospital, Crewe Road South, Edinburgh EH4 2XU, UK. Tel: + 44131651 1014; Fax: + 44131651 1013;

E-mail: s.m.holloway@ed.ac.uk

Received 16 May 2007; revised 18 December 2007; accepted 16 January 2008; published online 20 February 2008 to $55 \%$, although over $75 \%$ of those attending for counselling proceed to testing. ${ }^{4}$

We investigated the uptake of genetic testing by members of families with a BRCA1/2 mutation resident in South East Scotland. We further established the relationship between rate of uptake and sex, affected status, presence of children, degree of relationship to the index case, age and socioeconomic deprivation.

\section{Families}

The South East of Scotland Clinical Genetics Service serves a population of 1.2 million, resident in the Lothian, Borders and South West Fife regions of Scotland. Affected and unaffected members of families, with a history of breast and/or ovarian cancer, are referred by General Practitioners (GPs) and hospital consultants for genetic 
counselling and genetic testing if appropriate. For some patients, a BRCA1/2 mutation has already been identified in a family member. For other patients, genetic testing, of a family member affected with breast and/or ovarian cancer, is suggested at the appointment. By 1 April 2006, members of a total of 54 families in which a BRCA1 (31 families) or a BRCA2 (23 families) mutation had been identified (before or after referral but before 1 January 2005) had been counselled. We investigated the uptake of genetic testing by relatives of the index case in these families. The index case was defined as the first person to be identified as a mutation carrier. Relatives included in the study were resident in South East Scotland (Lothian, Borders and south west Fife regions).

At the genetic counselling appointment, the index case was asked to inform at-risk relatives that a gene conferring an increased risk of breast and ovarian cancer had been found in the family, that screening was available for these cancers and that if they wanted more information about genetic testing they should contact their local Genetics Centre or their GP. A few index cases requested copies of a letter containing the above statement to pass on to their relatives. If the index case was deceased or lived outside the area covered by the Clinical Genetics Service another family member, previously counselled by the Clinical Genetics Service, was informed of the results and asked to contact other relatives. Clinical Genetics staff did not, however, ask patients whether they had made contact with relatives or what information they had given to them. More recently, with the permission of the index case, family members, previously counselled by the Clinical Genetics Service, were contacted when a mutation was identified in their family to inform them of the possibility of testing.

Relatives of the index case, who made contact with the Genetics Service directly or via their GP, were invited to attend an appointment for genetic counselling at which the mutation findings were discussed and the offer of genetic testing made. Relatives wishing to be tested received their results 4-6 weeks after giving a blood sample. In the results given below, relatives are classified according to their testing status on 1 April 2006.

\section{Methods}

Attendance for genetic counselling and uptake of testing were recorded in different groups of relatives of the index case.

\section{Socioeconomic deprivation and attendance for genetic counselling after identification of the familial mutation}

The Carstairs Deprivation Score (CDS) for Scottish postcode sectors from the 2001 Census $^{5}$ was used as a measure of socioeconomic deprivation. Postcodes were not known for relatives who did not attend the Clinical Genetics Service. We therefore obtained the CDS for the index case or, if the index case had not attended the Clinical Genetics Service, the score for the first person to attend in the family. This locality-based score was used as a measure of deprivation for each family. The $\chi^{2}$ test was used to compare the numbers attending for counselling in different groups.

\section{Time interval between identification of the familial mutation and test results}

The time interval between identification of the mutation in the index case and the relative's test result was defined as the number of months between the dates on the laboratory reports giving the two results. Differences between groups were analysed using the Mann-Whitney $U$ test. Since all relatives in this study were from families with a known mutation, we assumed that there was a uniform delay in obtaining the relative's result after the decision to undergo testing was made. Thus, the time interval described above was taken as a measure of the time taken by the relative to make a decision about whether to undergo testing.

\section{Relatives counselled by the Genetics Service and their decision to undergo testing}

Comparisons were made between the numbers of counselled relatives who differed in sex, affected status, presence of offspring and degree of relationship to the index case, who underwent testing using the $\chi^{2}$ test (or Fisher's exact test). Relatives were classified as being affected if clinical records indicated that they had had a diagnosis of breast and/or ovarian cancer. The $t$-test was used to compare the tested and untested relatives with respect to age of the relative on the date of the laboratory report for the result in the index case and CDS score for their own postcode of residence.

Multiple logistic regression was used to estimate simultaneously the relationship of each of the above six variables to the probability of membership of the tested group.

All calculations were made using SPSS version 14.0.

\section{Results}

Families

Index cases The number of index cases resident in South East Scotland was 25 (46\%), 11 (20\%) were resident in other parts of Scotland, 11 (20\%) in other parts of the UK and 7 (13\%) overseas. The familial mutation was identified in the years 1996-1999 in 13 (24\%) carriers and in the years 2000-2004 in 41 (76\%). The median time period since the mutation was identified was 37 months.

Relatives of index cases at risk of carrying the gene mutation Numbers of relatives of the index case resident in South East Scotland at risk of carrying the gene mutation 
Table 1 Numbers of relatives resident in South East Scotland at risk of carrying the gene mutation at the time it was identified

\begin{tabular}{lrrr}
\hline & \multicolumn{3}{c}{ Sex } \\
Relative & Male & Female & Total \\
\hline First degree $\geq 18$ years & 40 & 69 & 109 \\
Second degree and more distant $\geq 18 y e a r s$ & 85 & 118 & 203 \\
Relatives $<18$ years & 66 & 55 & 121 \\
Total & 191 & 242 & 433 \\
\hline
\end{tabular}

(at the time it was identified) were calculated from recorded family pedigrees (Table 1 ). Among the second and more distant relatives, 158 (78\%) were second- and third-degree relatives with the remainder being mainly more distant relatives (in families where closer relatives were deceased or resident outside South East Scotland). There were approximately six adult relatives and two child relatives per index case resident in South East Scotland.

Attendance for genetic counselling and uptake of testing First-degree relatives Details of attendance for genetic counselling and uptake of testing in first-degree relatives are given in Figure 1 and summarised in Table 2 . The percentage of relatives tested was 39\% (8\% of males and $58 \%$ of females).

Second-degree and more distant relatives We identified 203 relatives eligible for testing at the time the index case received their result but 43 (17 male relatives, 26 female relatives) were no longer at risk of being mutation carriers by April 2006 because an ancestor had tested negative for the mutation. These relatives were not included in any of the calculations, which follow. Details of uptake of testing in the remaining 160 relatives are given in Figure 1 and summarised in Table 2 . The percentage of relatives tested was $26 \%$ (9\% of males and $39 \%$ of females).

All relatives The number of relatives who attended for genetic counselling was 117 (43\%) (17 (16\%) male relatives, $100(62 \%)$ female relatives). Of those relatives counselled, 85 (73\%) (9 (53\%) male relatives counselled and $76(76 \%)$ female relatives counselled) were tested. Thus, a total of $85(32 \%)$ (9 (8\%) male, 76 (47\%) female) eligible relatives in our families were tested.

Socioeconomic deprivation and attendance for genetic counselling after identification of the familial mutation Six of the index cases in the 54 families had no relatives in South East Scotland. The CDS for the other families ranged between -5.22 (least deprived family) and 2.68 (most deprived family). The mean score was -1.2. Families were classified according to whether their CDS was $<-1.2$ (23 families, less deprived) or $>-1.2$ (25 families, more deprived). Table 3 shows the total numbers of relatives in these families by whether counselled and/or tested and by degree of relationship to the index case and deprivation group. Comparisons of the numbers who had and had not received counselling were made between the two groups of first-degree and the two groups of seconddegree and more distant relatives. There was a significant $(P<0.05)$ difference between the two deprivation groups in the numbers of first-degree relatives attending for counselling. The numbers were 21 (67.7\%, 95\% CL 50.1-81.4\%) of the least deprived group and 32 (46.4\%, 95\% CL 35.1$58.0 \%$ ) of the more deprived group. There was no difference among more distant relatives.

Time interval between identification of the familial mutation and test results For first-degree relatives, the time interval ranged from 1 to 56 months with a median of 7 months (interquartile range 3-14 months). For more distant relatives, the time interval was significantly $(P<0.01)$ longer with a range of $2-61$ months and median of 14 months (interquartile range $7-23$ months).

Relatives counselled by the genetics service and their decision to undergo testing The number of relatives who underwent testing was 85 with 32 declining testing. Numbers and percentages of different categories of relative undergoing testing are given in Table 4 . Relatives were significantly more likely to be tested if they were female, affected or had children. First-degree relatives were more likely to be tested than more distant relatives but the difference was nor statistically significant.

Relatives opting for testing were older when the mutation was identified (mean $( \pm S D): 41.9 \pm 10.4$ years) than relatives who declined testing (mean $( \pm S D)$ : $36.8 \pm 12.7$ years) $(P<0.05)$. There were no significant differences between the groups in socioeconomic deprivation score (mean CDS was -0.8 (95\% CL -1.3 to -0.2$)$ in the tested group and $-1.2(95 \% \mathrm{CL}-2.3$ to -0.2$)$ in the group not tested).

When logistic regression analysis was carried out using all the above variables none of the regression coefficients were significant.

\section{Discussion}

The overall uptake of testing among eligible relatives was $32 \%$ and $73 \%$ of relatives attending for genetic counselling proceeded to testing. Over $50 \%$ of eligible relatives never made contact with the Genetics Service. These rates are very similar to those reported for a Manchester Clinic. ${ }^{4}$ In both studies, family members were asked to inform relatives of the availability of genetic testing. Relatives who did not make contact may have simply been unaware of the possibility of testing rather than deciding against it. Since patients were not asked whether they had informed 

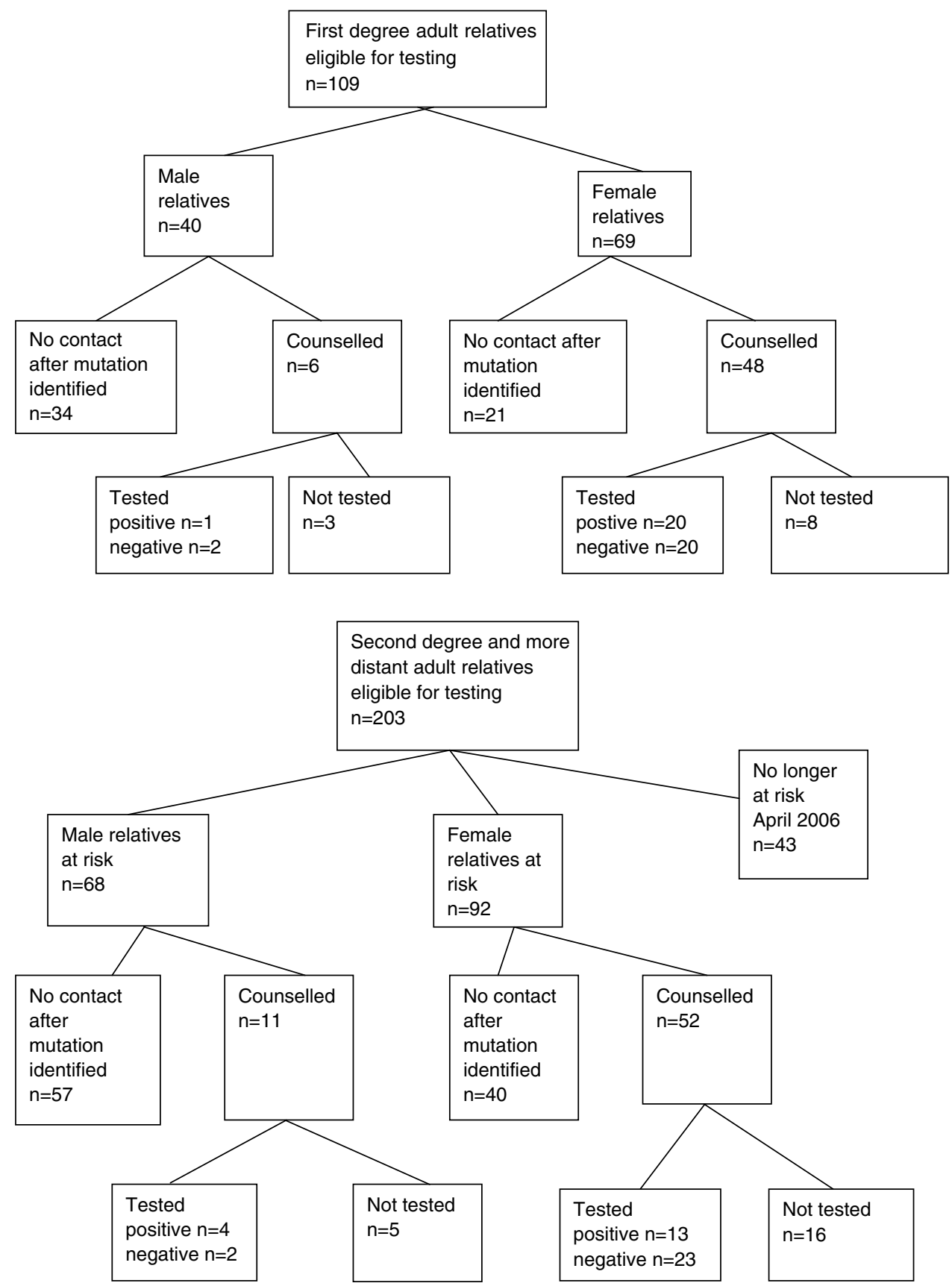

Figure 1 Diagram showing numbers of relatives of index cases eligible for testing and numbers counselled and tested.

their relatives of the possibility of testing, we are unable to analyse communication patterns and decision-making processes in more detail. However, the percentage of firstdegree relatives who received genetic counselling was greater than that in more distant relatives. In a study in France, ${ }^{6}$ in which the index cases had known of their mutation status a shorter time than in our study, there was a greater difference between the attendance rate for genetic counselling in first and second-degree relatives. In both of these studies and in other similar studies, ${ }^{4,7}$ there was no significant difference in the percentages of first-degree and more distant relatives who subsequently received genetic testing. These findings suggest that differences in uptake by closer and more distant relatives are likely to be due to the time required to disseminate the information among family members. Ignorance of the possibility of testing may be one of the causes of low uptake of genetic testing especially soon after identification of the familial 
Table 2 Numbers (\%) of first-degree and second-degree and more distant relatives by whether counselled and/or tested

\begin{tabular}{|c|c|c|c|c|}
\hline \multirow[b]{2}{*}{ Degree of relative } & \multirow[b]{2}{*}{ Whether counselled or tested } & \multicolumn{2}{|c|}{ Sex } & \multirow[b]{2}{*}{ Total } \\
\hline & & Male & Female & \\
\hline First degree & $\begin{array}{l}\text { Counselled and tested } \\
\text { Counselled not tested } \\
\text { No contact } \\
\text { Total }\end{array}$ & $\begin{array}{c}3(7.5 \%) \\
3(7.5 \%) \\
34(85.0 \%) \\
40(100.0 \%)\end{array}$ & $\begin{array}{c}40(58.0 \%) \\
8(11.6 \%) \\
21(30.4 \%) \\
69(100.0 \%)\end{array}$ & $\begin{array}{l}43(39.4 \%) \\
11(10.1 \%) \\
55(50.5 \%) \\
109(100.0 \%)\end{array}$ \\
\hline Second degree and more distant & $\begin{array}{l}\text { Counselled and tested } \\
\text { Counselled not tested } \\
\text { No contact } \\
\text { Total }\end{array}$ & $\begin{array}{c}6(8.8 \%) \\
5(7.4 \%) \\
57(83.8 \%) \\
68(100.0 \%)\end{array}$ & $\begin{array}{l}36(39.1 \%) \\
16(17.4 \%) \\
40(43.5 \%) \\
92(100.0 \%)\end{array}$ & $\begin{array}{c}42(26.3 \%) \\
21(13.1 \%) \\
97(60.6 \%) \\
160(100.0 \%)\end{array}$ \\
\hline
\end{tabular}

Table 3 Numbers (\%) of relatives in families in different socioeconomic deprivation groups by whether counselled and/or tested after the familial mutation was identified

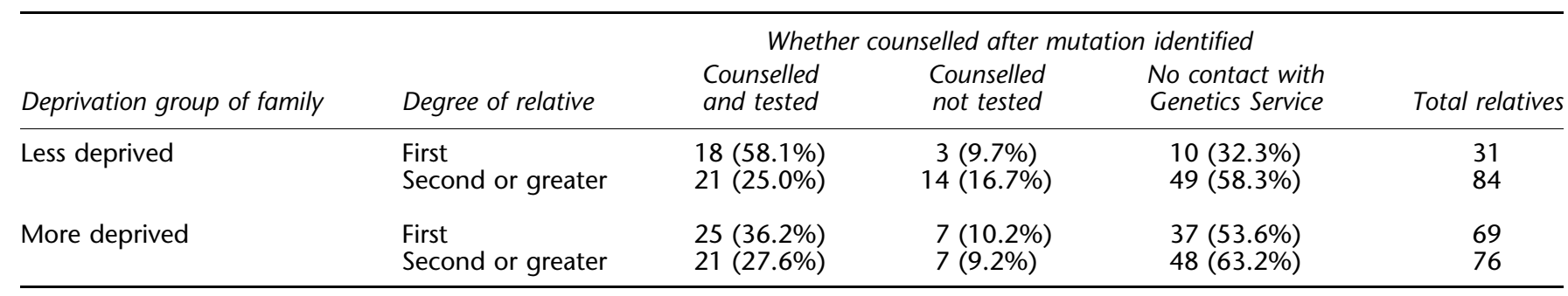

Table 4 Number (\%) of different categories of counselled relatives tested

\begin{tabular}{|c|c|c|}
\hline Category of relative & Number & Percent (95\% Confidence interval) \\
\hline \multicolumn{3}{|l|}{$\operatorname{Sex}^{*}$} \\
\hline Male & 9 & $52.9(31.0-73.8 \%)$ \\
\hline Female & 76 & $76.0(66.8-83.3 \%)$ \\
\hline \multicolumn{3}{|l|}{ Affected status* (female only) } \\
\hline Affected & 13 & $100(77.2-100.0 \%)$ \\
\hline Unaffected & 63 & $72.4(62.2-80.7 \%)$ \\
\hline At least 1 child & 66 & $81.5(71.7-88.4 \%)$ \\
\hline No children & 19 & $52.8(37.0-68.0 \%)$ \\
\hline \multicolumn{3}{|l|}{ Relationship to index case } \\
\hline First degree & 43 & $79.6(67.1-88.2 \%)$ \\
\hline Second degree or greater & 42 & $66.7(54.4-77.1 \%)$ \\
\hline
\end{tabular}

${ }^{*} P<0.05,{ }^{* *} P<0.001$.

mutation. In support of this are the findings of a study of communication of test results in families with Hereditary Non Polyposis Colorectal Cancer (HNPCC) ${ }^{8}$ in which participants felt primarily responsible for notifying members of their nuclear family (i.e. siblings and children), rather than more distant relatives.

All relatives included in the study were resident in South East Scotland and could contact the Clinical Genetics Service directly or via their GP to request counselling. Attendance for genetic counselling in at-risk family members is likely to be influenced by the quality of information given by a relative. A few patients requested written information to pass on to relatives. For this purpose, they were given a letter giving information about the inheritance of breast and ovarian cancer in the family, screening for these cancers and the possibility of genetic testing. In other studies, ${ }^{4,9}$ similar written information was available for index cases to distribute among their relatives but practice seems to vary between Services. However, the majority of relatives in our study only received verbal information. If this information was inaccurate or not easily understandable relatives might be less likely to seek genetic advice. 
The greater time interval between identification of the mutation in the index case and the relative's test result in more distant compared to first-degree relatives is at least partly because more distant relatives may wait for a result in a closer relative before deciding whether to undergo testing.

Attendance for counselling among first-degree relatives was lower in more socioeconomically deprived families, but there was no association between uptake of testing and socioeconomic deprivation among all relatives counselled. In some studies, ${ }^{10,11}$ interest in genetic testing was associated positively with better education, whereas in other studies $^{12,13}$ there was no significant association and in another study ${ }^{14}$ there was a negative association. These differences are likely to be due to differences in study design and the patient groups involved. In a study ${ }^{15}$ of families with HNPCC, it was suggested that individuals with less education might have less understanding of the concept of genetic testing. In a Canadian study, ${ }^{16}$ reasons given by women for being interested in genetic testing for breast cancer indicated a lack of understanding about the limitations and implications of testing. These findings indicate that there is a need for medical professionals to provide information for family members, which is easily understandable to persons of different socioeconomic backgrounds. The lack of an association between attendance for counselling and socioeconomic deprivation in more distant relatives may be due to the use of the CDS of the index case as a measure of family deprivation. More distant relatives are more likely to differ in this respect from the index case.

About $70 \%$ of relatives received their result within 9 months of identification of the familial mutation, which was similar to the findings of a Dutch study. ${ }^{9}$ However, $15 \%$ of first-degree relatives waited over 2 years to be tested, suggesting that relatives often need time to make a decision about testing. In the Manchester study, ${ }^{4} 27 \%$ of all relatives did not undergo testing until at least 2 years after the family was first informed.

We found that the percentage of eligible females who underwent testing was six times higher than that of males. Among relatives who underwent counselling the ratio was 1.5. Brooks et $a l^{4}$ found similar percentages in their Manchester clinic. In another study, ${ }^{17}$ a greater percentage of females than of males was tested but the difference was not statistically significant. Brooks et $a l^{4}$ felt that the difference between the sexes in uptake of testing was likely to be due to poor communication within families. The difference between the sexes in uptake after counselling suggests that males may feel that the test is less relevant to them because of its principal association with the risk of developing breast and ovarian cancer. In a study of communication patterns of BRCA1/2 test results, ${ }^{18}$ participants shared their result more often with female than male first-degree relatives. It was suggested that the mutation carrier underestimated the desire of their male relatives to be aware of the familial mutation and that materials should be developed specifically targeting men.

Only a few counselled female relatives were affected but they were more likely to undergo testing than unaffected female relatives. These findings are in agreement with those of another study, ${ }^{17}$ but in two other studies ${ }^{6,7}$ there was no difference in uptake by counselled affected and unaffected relatives. Affected relatives may be more likely to undergo testing for the sake of other family members since, as they already have cancer, a positive result may be less devastating to them than to a healthy relative.

Parenthood was found to be a significant predictor of utilisation of testing in our study and also in a study in the Netherlands $^{9}$ where the effect was greater in men than women. The number of tested males in our study was small but $78 \%$ of these had children compared with $40 \%$ of untested males.

In our study and in two other studies, ${ }^{7,19}$ older relatives were more likely to be tested than younger relatives, whereas in another study ${ }^{9}$ younger women (but not men) were more likely to undergo testing than older women (men). Older relatives may be more interested in testing because they are at an age when they are more likely to develop cancer. Alternatively, younger people are more likely to be working and their busy lives may make clinic attendance more difficult. They may prefer to wait until they are at an age when clinical surveillance would be initiated.

Individuals identified to be gene carriers can start a cascading process. Cascading might identify gene carriers who would otherwise not be eligible for breast cancer screening on family history or clinical grounds alone. This is particularly relevant for female descendants of male gene carriers who in turn inherited the BRCA1/2 gene mutation through the male line. In contrast, a negative test result reduces unnecessary and potentially harmful breast screening procedures for the tested individual. It also signifies that no further mutation transmission is possible in this particular branch of the family and removes the need for breast cancer screening and unnecessary anxiety for all their descendants. Cascade screening is the most costeffective way of identifying individuals within the population with BRCA1/2 mutations.

The main limitation of this study is that the findings are based upon only 54 families resident in South East Scotland. Only 117 relatives of the index cases were counselled. Thus, the lack of a statistically significant difference in some group comparisons may have been due to small numbers. No information was available to us about relatives outside South East Scotland. Their inclusion might have altered the results of our study if they had differed from the other relatives in the factors considered. Despite these limitations, however, it is of interest that our results are similar to those of other studies. 


\section{Conclusions}

The results of this study show that despite earlier predictions of a high uptake of genetic testing by relatives in breast/ovarian cancer families, the actual number of at-risk individuals opting for genetic testing is much smaller than anticipated. There is some evidence that this is partly due to lack of communication or delayed communication within families especially soon after the mutation is identified. Attendance figures for genetic counselling and testing in at-risk male family members remain unsatisfactory. Effective communication strategies targeted at male family members need to be developed to improve awareness. Inequalities of use of Genetics Services by different socioeconomic groups are a further matter of concern. This may be partly the result of lack of understanding of the testing process in families from more deprived backgrounds. Cancer morbidity and mortality in families with BRCA1/2 mutations can only be reduced if all potential gene carriers are offered information about their genetic testing options. A better understanding of family communication may lead to more effective counselling strategies. Dissemination of relevant information might be improved by maximising the mutation carrier's ability to be a competent and confident informant. This could be achieved by provision of educational materials specifically targeted towards relatives, by improved support for mutation carriers, for example, through a discussion about communication strategies and an increased emphasis on the importance of testing both sexes. Care pathways could unify and streamline counselling practice.

\section{References}

1 Lerman C, Seay J, Balshem A, Audrain J: Interest in genetic testing among first-degree relatives of breast cancer patients. Am J Med Genet 1995; 57: 385-392.

2 Struewing JP, Lerman C, Kase RG, Giambarresi TR, Tucker MA: Anticipated uptake and impact of genetic testing in hereditary breast and ovarian cancer families. Cancer Epidemiol Biomarkers Prev 1995; 4: 169-173.

3 Jacobsen PB, Valdimarsdottier HB, Brown KL, Offit K: Decisionmaking about genetic testing among women at familial risk for breast cancer. Psychosom Med 1997; 59: 459-466.

4 Brooks L, Lennard F, Shenton A et al: BRCA1/2 predictive testing: a study of uptake in two centres. Eur J Hum Genet 2004; 12: $654-662$.
5 McLoone P: Carstairs scores for Scottish postcode sectors from the 2001 Census. MRC Social and Public Health Sciences Unit. University of Glasgow: Glasgow, 2004.

6 Julian-Reynier C, Sobol H, Sévilla C, Noguès C, Bourret P, The French Cancer Genetic Network: Uptake of hereditary breast/ ovarian cancer genetic testing in a French national sample of BRCA1 families. Psychooncology 2000; 9: 504-510.

7 Biesecker BB, Ishibe N, Hadley DW et al: Psychosocial factors predicting BRCA1/BRCA2 testing decisions in members of hereditary breast and ovarian cancer families. Am J Med Genet 2000; 93: 257-263.

8 Peterson SK, Watts BG, Koehly LM et al: How families communicate about HNPCC genetic testing: findings from a qualitative study. Am J Med Genet 2003; 119C: 78-86.

9 Meijers-Heijboer EJ, Verhoog LC, Brekelmans CT et al: Presymptomatic DNA testing and prophylactic surgery in families with a BRCA1 or BRCA2 mutation. Lancet 2000; 355: 2015-2020.

10 Lerman C, Daly M, Masny A, Balshem A: Attitudes about genetic testing for breast-ovarian cancer susceptibility. J Clin Oncol 1994; 12: $843-850$.

11 Armstrong K, Micco E, Carney A, Stopfer J, Putt M: Racial differences in the use of BRCA1/2 testing among women with a family history of breast and ovarian cancer. JAMA 2005; 293: $1729-1736$.

12 Meiser B, Butow P, Barratt A et al: Attitudes to genetic testing for breast cancer susceptibility in women at increased risk of developing hereditary breast cancer. I Med Genet 2000; 37: $472-476$.

13 Gwyn K, Vernon SW, Conoley PM: Intention to pursue genetic testing for breast cancer among women due for screening mammography. Cancer Epidemiol Biomarkers Prev 2003; 12: 96-102.

14 Kash KM, Ortega-Verdejo K, Dabney MK, Holland JC, Miller DG, Osborne MP: Psychosocial aspects of cancer genetics: women at high risk for breast and ovarian cancer. Semin Surg Oncol 2000; 18: 333-338.

15 Lerman C, Hughes C, Trock BJ et al: Genetic testing in families with hereditary nonpolyposis colon cancer. JAMA 1999; 281: $1618-1622$.

16 Bottorff JL, Ratner PA, Balneaves LG et al: Women's interest in genetic testing for breast cancer risk: the influence of sociodemographics and knowledge. Cancer Epidemiol Biomarkers Prev 2002; 11: 89-95.

17 Keogh LA, Southney MC, Maskiell J et al: Uptake of offer to receive genetic information about BRCA1 and BRCA2 mutations in an Australian population-based study. Cancer Epidemiol Biomarkers Prev 2004; 13: 2258-2263.

18 Patenaude AF, Dorval M, DiGianni LS, Schneider KA, Chittenden A, Garber JE: Sharing BRCA1/2 test results with first-degree relatives: factors predicting who women tell. J Clin Oncol 2006; 24: 700-706.

19 Foster C, Evans DG, Eeles R et al: Non uptake of predictive genetic testing for BRCA1/2 among relatives of known carriers: attributes, cancer worry, and barriers to testing in a multicenter clinical cohort. Genet Test 2004; 8: 23-29. 